\title{
Superconductivity in the Niobium-rich compound $\mathrm{Nb}_{5} \mathrm{Se}_{4}$
}

\author{
T. Klimczuk ${ }^{1}$, K. Baroudi ${ }^{2}$, J.W. Krizan ${ }^{2}$, A.L. Kozub ${ }^{1}$, and R.J. Cava ${ }^{2}$ \\ ${ }^{1}$ Faculty of Applied Physics and Mathematics, Gdansk University of Technology, \\ Narutowicza 11/12, 80-233 Gdansk, Poland \\ ${ }^{2}$ Department of Chemistry, Princeton University, Princeton NJ 08544, USA
}

\begin{abstract}
The niobium rich selenide compound $\mathrm{Nb}_{5} \mathrm{Se}_{4}$ was synthesized at ambient pressure by high-temperature solid-state reaction in a sealed Ta tube. Resistivity and heat capacity measurements reveal that this compound is superconducting, with a $\mathrm{T}_{\mathrm{c}}=1.85 \mathrm{~K}$. The electronic contribution to the specific heat $\gamma$ and the Debye temperature are found to be $18.1 \mathrm{mJmol}^{-1} \mathrm{~K}^{-2}$ and $298 \mathrm{~K}$ respectively. The calculated electron-phonon coupling constant $\lambda_{\text {ep }}=0.5$ and the $\Delta \mathrm{C}_{\mathrm{p}} / \gamma \mathrm{T}_{\mathrm{c}}=1.42$ ratio imply that $\mathrm{Nb}_{5} \mathrm{Se}_{4}$ is a weak coupling BCS superconductor. The upper critical field and coherence length are found to be $1.44 \mathrm{~T}$ and $15.1 \mathrm{~nm}$, respectively.
\end{abstract}

Corresponding author: Tomasz Klimczuk (e-mail: tomasz.klimczuk@pg.gda.pl) 


\section{Introduction}

Although it is a poor metal, niobium holds the record for the highest superconducting critical temperature $\left(\mathrm{T}_{\mathrm{c}}\right)$ among elements under ambient pressure. Its remarkably high $\mathrm{T}_{\mathrm{c}}, 9.2 \mathrm{~K}$, influenced physicists to search for superconducting alloys in the 1960s and resulted in finding the now well-known $\mathrm{NbTi}$ and $\mathrm{Nb}_{3} \mathrm{Sn}$ superconductors. From an empirical perspective, one can therefore propose that metallic compounds containing $\mathrm{Nb}$ in significant proportion are good candidates for superconductivity. Of particular interest due to the fact that they can display electronic ground states that compete with superconductivity are compounds of $\mathrm{Nb}$ with the chalcogenide element Se. There are nine binary $\mathrm{Nb}-\mathrm{Se}$ compounds reported in the literature, displaying a variety of physical properties: charge-density-wave (CDW) formation in $\mathrm{NbSe}_{2}[1,2]$ and $\mathrm{NbSe}_{3}$ [3, 4], semiconducting behavior in $\mathrm{Nb}_{2} \mathrm{Se}_{9}$ [5], and superconductivity in $\mathrm{Nb}_{3} \mathrm{Se}_{4}$ [6] and $\mathrm{NbSe}_{2}[7,8]$. The coexistence of multigap superconductivity, $\mathrm{T}_{\mathrm{c}} \sim 7 \mathrm{~K}$, with $\mathrm{CDW}$ formation, $\mathrm{T}_{\mathrm{CDW}}=35 \mathrm{~K}$ [e.g. refs. 1, 2], makes $\mathrm{NbSe}_{2}$, for instance, a particularly interesting material.

In contrast to the case for $\mathrm{NbSe}_{2}$, there are not many reports focused on the physical properties of metal rich (i.e. a compound with more metal atoms than nonmetal atoms) niobium selenides. This is likely caused by the high temperature required for the synthesis of these compounds and the volatility of elemental Se. K. Tsukuma et. al [9] studied the $\mathrm{Nb}_{5} \mathrm{Se}_{4-\mathrm{x}} \mathrm{S}_{\mathrm{x}}$ system synthesized at high pressures. The properties of the binary compound $\mathrm{Nb}_{5} \mathrm{Se}_{4}$ were not reported, but resistivity measurements on a high-pressure-synthesized sample with composition $\mathrm{Nb}_{5} \mathrm{Se}_{2} \mathrm{~S}_{2}$ showed a superconducting transition at $3.4 \mathrm{~K}$. Here we report the high temperature synthesis, at ambient pressure, of the metal rich niobium selenide $\mathrm{Nb}_{5} \mathrm{Se}_{4}$. We find that this compound is superconducting at $1.85 \mathrm{~K}$. Characterization of the superconductivity of $\mathrm{Nb}_{5} \mathrm{Se}_{4}$ indicates that it is a weak coupling BCS superconductor. We compare its superconducting properties to those of elemental $\mathrm{Nb}_{3} \mathrm{Se}_{4}, \mathrm{NbSe}_{2}$ and $\mathrm{Nb}_{5-\delta} \mathrm{Te}_{4}$. 


\section{Experimental}

A polycrystalline sample of $\mathrm{Nb}_{5} \mathrm{Se}_{4}$ was synthesized by high-temperature solidstate reaction from $\mathrm{NbSe}_{2}$ and elemental $\mathrm{Nb}$. The $\mathrm{NbSe}_{2}$ precursor was prepared by heating $\mathrm{Nb}$ powder (Alfa Aesar 99.9\%) and Se (Alfa Aesar 99.999\%) in an evacuated quartz tube at $870 \mathrm{~K}$ for 5 hours followed by heating at $1020 \mathrm{~K}$ for 36 hours. The powder of the precursor was determined to be pure by the x-ray diffraction technique. $\mathrm{NbSe}_{2}$ was then mixed with $\mathrm{Nb}$ powder in the appropriate stoichiometric ratio, thoroughly ground, pelletized under a pressure of $300 \mathrm{MPa}$, and sealed in a $\mathrm{Ta}$ ampoule in a high purity Ar atmosphere. The ampoule was subsequently heated at 1620 for 5 hours in a vacuum furnace (Materials Research Furnaces, Inc.). This formed the $\mathrm{Nb}_{5} \mathrm{Se}_{4}$ compound. To anneal out possible defects, the pellet was then heated in an evacuated sealed quartz glass tube at $1170 \mathrm{~K}$ for 12 hours. Powder X-ray diffraction (PXRD, Bruker D8 Focus, $\mathrm{Cu} K_{\alpha}$ radiation, graphite diffracted beam monochromator) was used to structurally characterize the sample by the Rietveld method [10] through use of the FullProf 5.30 program [11]. Measurements of the temperature dependence of the electrical resistivity and heat capacity were performed in a Quantum Design Physical Property Measurement System (PPMS) equipped with a ${ }^{3}$ He cryostat.

\section{Results}

The room temperature powder X-ray diffraction (PXRD) pattern for the $\mathrm{Nb}_{5} \mathrm{Se}_{4}$ sample, with a successful structural fit of the data to the $\mathrm{Ti}_{5} \mathrm{Te}_{4}$ structure type, reported previously for $\mathrm{Nb}_{5} \mathrm{Se}_{4}$ [12] is shown in Fig. 1. The excellent quality of the refinement and the absence of additional reflections in the PXRD pattern confirm the high purity of the $\mathrm{Nb}_{5} \mathrm{Se}_{4}$ sample. The estimated lattice parameters and the $8 h$ position coordinates obtained in the refinement are close to those reported by Selte and Kjekshus [12]. The refined structural parameters are summarized in Table I.

A structural model with niobium vacancies present was also tested, in analogy to what is found for the nonstoichiometric compound $\mathrm{Nb}_{5-\delta} \mathrm{Te}_{4}$ (ref. 13). This model did not result in a significant change to the already excellent fit. This is commensurate with the difference in synthetic procedures for these two materials. Synthesizing $\mathrm{Nb}_{5} \mathrm{Se}_{4}$ as a pellet in a sealed tantalum tube does not offer a mechanism for the bulk sample to be niobium poor. The tantalum tube was clean and did not show any sign of 
a chemical attack, and thus the presence of an elemental niobium metal impurity, as would be required for a matter-conserving synthesis if the $\mathrm{Nb}_{5} \mathrm{Se}_{4}$-type phase is $\mathrm{Nb}$ deficient, would be readily detectable in the powder diffraction pattern of the bulk material and through the presence of a superconducting transition at the $T_{c}$ of $\mathrm{Nb}$, neither of which is observed. Given the excellent fit of the diffraction data to the ideal structure model, and the lack of any other evidence for nonstoichiometry, the composition of the phase is well established.

$\mathrm{Nb}_{5} \mathrm{Se}_{4}$ has a tetragonal, body-centered crystal structure, based on square prisms of niobium atoms, with short edges of $3.14 \AA$ in the plane and long edges of $3.45 \AA$ along the $c$ axis. The prisms share square faces to form infinite chains along $c$ (inset of Figure 1). Each prism has another $\mathrm{Nb}$ atom in its center, such that the structure can be considered as consisting of chains of body centered, stretched $\mathrm{Nb}$ cubes sharing faces along $c$, separated by Se atoms. Alternatively, the crystal structure of $\mathrm{Nb}_{5} \mathrm{Se}_{4}$ can be seen as infinite chain of compressed $\mathrm{Nb}_{6} \mathrm{Se}_{8}$ clusters [13].

The temperature dependence of the electrical resistivity $\rho(\mathrm{T})$ of $\mathrm{Nb}_{5} \mathrm{Se}_{4}$ from $0.5 \mathrm{~K}$ to $300 \mathrm{~K}$, measured in zero applied magnetic field, is presented in the main panel of Figure 2. The $\rho(\mathrm{T})$ data demonstrates a positive derivative $(\mathrm{d} \rho / \mathrm{dT}>0)$ and a residual resistivity ratio RRR slightly less than 2, which is typical for a metal in a polycrystalline form where grain boundaries conduct poorly [14]. The red line through the experimental data represents a fit that combines a Bloch-Grüneisen resistivity $\rho_{\mathrm{BG}}$ together with a parallel resistor $\rho_{\mathrm{p}}$ :

$$
\begin{aligned}
& \rho(T)^{-1}=\rho_{P}^{-1}+\left(\rho_{0}+\rho_{B G}\right)^{-1} \text {, where } \\
& \rho_{B G}=4 \mathrm{R} \Theta_{\mathrm{R}}\left(\frac{T}{\Theta_{R}}\right)^{5} \int \frac{x^{5}}{(\exp (x)-1)(1-\exp (-x))} d x .
\end{aligned}
$$

The Bloch-Grüneisen fit to $\rho(T)$ gives: $\rho_{0}=2.8 \mathrm{~m} \Omega \mathrm{cm}, \rho_{P}=3.9 \mathrm{~m} \Omega \mathrm{cm}$, and $\Theta_{R}=$ $182 \mathrm{~K}$. Low temperature resistivity data measured under magnetic field from $0 \mathrm{~T}$ to 1 $\mathrm{T}$ is shown in the inset of Figure 2. In the zero field measurement, the onset of the superconducting transition is $\mathrm{T}_{\mathrm{c} \text { onset }} \sim 2 \mathrm{~K}$ and a sharp superconducting transition width of about $70 \mathrm{mK}$ (using the $90 \%$ - $10 \%$ criterion) is observed. The superconducting temperature $\left(\mathrm{T}_{\mathrm{c}}\right)$, defined as the temperature at which the resistivity dropped by $50 \%$ from the normal state, was estimated for each applied magnetic field. These data are shown in Figure 4 and will be discussed in the following. 
The characterization of the superconducting properties of $\mathrm{Nb}_{5} \mathrm{Se}_{4}$ by specific heat is shown in Figure 3. Panel (a) shows the temperature dependence of $\mathrm{C}_{\mathrm{p}} / \mathrm{T}$ at magnetic fields from $\mu_{0} \mathrm{H}=0$ to 0.8 Tesla (T). A sharp $\lambda$ - type anomaly confirms the presence of bulk superconductivity in $\mathrm{Nb}_{5} \mathrm{Se}_{4}$. The superconducting transition is suppressed to $\sim 0.7 \mathrm{~K}$ with the application of a field of $\mu_{0} \mathrm{H}=0.8 \mathrm{~T}$. The superconducting transition under zero magnetic field is presented in panel (b). The entropy conserving construction is shown by solid lines. Based on this construction, the superconducting critical temperature $\left(\mathrm{T}_{\mathrm{c}}=1.85 \mathrm{~K}\right)$ and the superconductivity jump $\left(\Delta \mathrm{C} / \mathrm{T}_{\mathrm{c}}=25.8 \mathrm{~mJ} \mathrm{~mol}^{-1} \mathrm{~K}^{-2}\right)$ were estimated. The $\mathrm{T}_{\mathrm{c}}$ obtained from the heat capacity measurements is very close to the value obtained by the resistivity method $\left(\mathrm{T}_{\mathrm{c}}=1.79\right.$ $K)$. The heat capacity, $\left(C_{p} / T\right)$ versus $T^{2}$, measured under a magnetic field $\mu_{0} H=2 \mathrm{~T}$, which exceeds the upper critical field for $\mathrm{Nb}_{5} \mathrm{Se}_{4}$, is presented in Figure 3(c). The data are fitted by the formula $C_{p} / T=\gamma+\beta T^{2}$, with the fit result represented by a red solid line. The Sommerfeld parameter (electronic specific heat coefficient) $\gamma=18.1 \mathrm{~mJ} \mathrm{~mol}^{-}$ ${ }^{1} \mathrm{~K}^{-2}$, and phonon specific heat coefficient $\beta=0.658 \mathrm{~mJ} \mathrm{~mol}^{-1} \mathrm{~K}^{-4}$ are extracted from these data. The Debye temperature estimated from $\beta$ by using $\Theta_{D}=\left(12 \pi^{4} n R / 5 \beta\right)^{1 / 3}$ is $\Theta_{\mathrm{D}}=298(1) \mathrm{K}$.

Knowing the Sommerfeld parameter and the Debye temperature allows for the estimation of several superconducting parameters. The normalized specific heat jump value $\Delta \mathrm{C} / \gamma \mathrm{T}_{\mathrm{c}}$ was found to be 1.42 , which is almost exactly that expected for the Bardeen-Cooper-Schrieffer (BCS) weak-coupling value (1.43). Weak coupling superconductivity for $\mathrm{Nb}_{5} \mathrm{Se}_{4}$ is also suggested from the electron-phonon constant value $\lambda_{\mathrm{ep}}=0.5$, obtained from the inverted McMillan equation:

$\lambda_{e p}=\frac{1.04+\mu^{*} \ln \left(\frac{\theta_{D}}{1.45 T_{C}}\right)}{\left(1-0.62 \mu^{*}\right) \ln \left(\frac{\theta_{D}}{1.45 T_{C}}\right)-1.04}$. For this calculation we used the Coulomb repulsion constant $\mu^{*}=0.13$, which falls in the range of $0.1-0.15$ used in the literature. The same value was also used for example by Karki, et al. (Ref. 15). Having estimated $\lambda_{\text {ep }}$, the density of states at the Fermi energy (for both spin directions) was calculated by using the relation: $\operatorname{DOS}\left(E_{F}\right)=\frac{6 \gamma}{\pi^{2} k_{B}^{2}\left(1+\lambda_{e p}\right)}$. It was found that $\operatorname{DOS}\left(\mathrm{E}_{\mathrm{F}}\right)$ 
$=5.1$ states $/ \mathrm{eV}$ f.u., very close to the reported $\operatorname{DOS}\left(\mathrm{E}_{\mathrm{F}}\right)=4.9$ states $/ \mathrm{eV}$ f.u. for $\mathrm{Nb}_{5-\delta} \mathrm{Te}_{4}$ (ref. 13).

Figure 4 presents the temperature dependence of the upper critical field for $\mathrm{Nb}_{5} \mathrm{Se}_{4}$, with the data points taken from the heat capacity (open squares) and resistivity (closed circles) measurements. The heat capacity data can be reasonably fitted by the theoretical expectations of the single band Werthamer-HelfandHohenberg (WHH) model in the dirty limit [16]. In this model $\mathrm{H}_{\mathrm{c} 2}(0)$ is calculated from the formula given in terms of digamma functions $\ln \left(\frac{1}{t}\right)=\psi\left(\frac{1}{2}+\frac{-}{2 t}\right)-\psi\left(\frac{1}{2}\right)$, where $t=\frac{T}{T_{c}}$ is the reduced temperature and $\bar{h}=\frac{4 H_{c 2}}{\pi^{2}\left(-d H_{c 2} / d t\right)_{t=1}}$. From the fit we estimate $\mu_{0} \mathrm{H}_{\mathrm{c} 2}(0)=0.98 \mathrm{~T}$. This is not consistent with the resistivity measurement that show a $\mathrm{T}_{\mathrm{c}}=0.55 \mathrm{~K}$ under applied magnetic field of $1 \mathrm{~T}$; thus a different analysis of this data is required.

In contrast, the temperature dependence of $\mathrm{H}_{\mathrm{c} 2}(\mathrm{~T})$ obtained from the resistivity measurement is almost perfectly linear $\left(R^{2}=0.9998\right)$, with the negative slope $\mathrm{d}\left(\mu_{0} \mathrm{H}_{\mathrm{c} 2}\right) / \mathrm{dT}=-0.812(5) \mathrm{T} / \mathrm{K}$ and the intercept value $\mu_{0} \mathrm{H}_{\mathrm{c} 2}(0)=1.444(6) \mathrm{T}$. Such a linear behavior for $\mathrm{H}_{\mathrm{c} 2}(\mathrm{~T})$ is predicted for a spheroidal Fermi surface [17]. Considering both the heat capacity and resistivity measurements, it is more consistent with the present results than the WHH model. Taking $\mu_{0} \mathrm{H}_{\mathrm{c} 2}(0)=1.444 \mathrm{~T}$, the superconducting coherence length for $\mathrm{Nb}_{5} \mathrm{Se}_{4}$ can be calculated by using the Ginzburg-Landau formula $\xi_{\mathrm{GL}}(0)=\left\{\phi_{0} /\left[2 \pi \mathrm{H}_{\mathrm{c} 2}(0)\right]\right\}^{1 / 2}=15.1 \mathrm{~nm}$. The superconducting properties of $\mathrm{Nb}_{5} \mathrm{Se}_{4}$ are compared to those of other niobium selenides in Table II.

\section{Conclusions}

In summary, we have successfully synthesized the niobium rich $\mathrm{Nb}_{5} \mathrm{Se}_{4}$ compound in high purity. Its crystal structure contains infinite chains of elongated body centered $\mathrm{Nb}$ cubes. In the normal state $\mathrm{Nb}_{5} \mathrm{Se}_{4}$ is a poor metal with $\mathrm{RRR} \sim 2$ and $\rho_{0}=1.6 \mathrm{~m} \Omega \mathrm{cm}$. The superconducting critical temperature, $\mathrm{T}_{\mathrm{c}}=1.85 \mathrm{~K}$, was determined by electrical resistivity and heat capacity measurements. The Sommerfeld

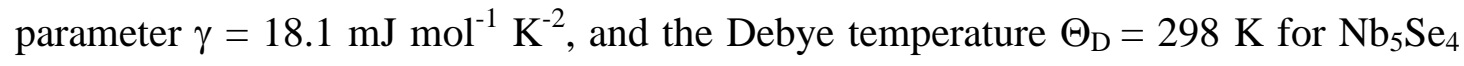


were estimated from the fit to the low temperature heat capacity data. The Debye temperature is higher than reported for $\mathrm{Nb}_{5-\delta} \mathrm{Te}_{4}$ (ref. 13) and reflects lower atomic mass of the Se atom. The normalized specific heat jump value $\Delta \mathrm{C} / \gamma \mathrm{T}_{\mathrm{c}}=1.42$ and the electron-phonon constant value $\lambda_{\text {ep }}=0.5$, suggest that $\mathrm{Nb}_{5} \mathrm{Se}_{4}$ is a BCS-type weak coupling superconductor.

The superconducting critical temperature, $\mathrm{T}_{\mathrm{c}}=1.85 \mathrm{~K}$, observed for $\mathrm{Nb}_{5} \mathrm{Se}_{4}$ is the lowest superconducting temperature among $\mathrm{Nb}$-Se binary compounds. The almost doubled $\mathrm{T}_{\mathrm{c}}=3.4 \mathrm{~K}$ observed for $\mathrm{Nb}_{5} \mathrm{Se}_{2} \mathrm{~S}_{2}$ [9], which has the same crystal structure as $\mathrm{Nb}_{5} \mathrm{Se}_{4}$, may be partially explained by replacement of half of the selenium by much lighter sulfur atoms in $\mathrm{Nb}_{5} \mathrm{Se}_{4-\mathrm{x}} \mathrm{S}_{\mathrm{x}}$. Similarly, the much lower $\mathrm{T}_{\mathrm{c}}$ observed for $\mathrm{Nb}_{5-\delta} \mathrm{Te}_{4}$ $\left(\mathrm{T}_{\mathrm{C}}=0.6-0.9 \mathrm{~K}\right.$, ref. 13) may be partially explained by the presence of the much heavier Te atom when compared to Se. Indeed, among three parameters that influence $\mathrm{T}_{\mathrm{c}}$ only the Debye temperature differs, whereas estimated $\lambda_{\mathrm{ep}}$ and $\mathrm{DOS}\left(\mathrm{E}_{\mathrm{F}}\right)$ for $\mathrm{Nb}_{5} \mathrm{Se}_{4}$ and $\mathrm{Nb}_{5-\delta} \mathrm{Te}_{4}$ are almost the same. The characterization of related materials with the same structure type would be of significant interest.

\section{Acknowledgments}

The materials synthesis and structural characterization performed at Princeton was supported by the US Department of Energy, grant DOE FG02-98ER45706. The research performed at the Gdansk University of Technology was financially supported by the National Science Centre (Poland) grant (DEC-2012/07/E/ST3/00584). 
Tables:

TABLE I

Refined structural parameters for $\mathrm{Nb}_{5} \mathrm{Se}_{4}$ at 298 K. Space group I 4/m (s.g. \# 87), $a=0.985247(23) \mathrm{nm}, c=0.345217(8) \mathrm{nm}$. Figures of merit: goodness of fit $\chi^{2}=1.84$, weighted profile residual $R_{w p}=18.9 \%$, profile residua; $R_{p}=17.1 \%$.

\begin{tabular}{|l|l|l|l|}
\hline \multicolumn{5}{|c|}{$\mathrm{Nb}_{5} \mathbf{S e}_{4}$} \\
\hline Atom & $\begin{array}{l}\text { Wyckoff } \\
\text { position }\end{array}$ & $\mathrm{x}$ & $\mathrm{Y}$ \\
\hline $\mathrm{Nb} 1$ & $2 \mathrm{a}(0,0,0)$ & 0 & 0 \\
\hline $\mathrm{Nb} 2$ & $8 \mathrm{~h}(\mathrm{x}, \mathrm{y}, 0)$ & $0.30437(16)$ & $0.37312(17)$ \\
\hline $\mathrm{Se}$ & $8 \mathrm{~h}(\mathrm{x}, \mathrm{y}, 0)$ & $0.05591(19)$ & $0.28292(19)$ \\
\hline
\end{tabular}

TABLE II. Superconducting parameters of $\mathrm{Nb}_{5} \mathrm{Se}_{4}$

\begin{tabular}{|l|l|l|l|l|l|}
\hline Parameter & Unit & $\mathrm{Nb}_{5} \mathrm{Se}_{4}{ }^{\mathrm{a}}$ & $\mathrm{Nb}_{3} \mathrm{Se}_{4}{ }^{\mathrm{b}}$ & $\mathrm{NbSe}_{2}{ }^{\mathrm{c}}$ & $\mathrm{Nb}_{5-\delta} \mathrm{Te}_{4}{ }^{\mathrm{d}}$ \\
\hline $\mathrm{T}_{\mathrm{c}}$ & $\mathrm{K}$ & 1.85 & 2.31 & 7.09 & $0.6-0.9$ \\
\hline$\Theta_{\mathrm{D}}$ & $\mathrm{K}$ & 298 & 220 & 236 & 259 \\
\hline$\gamma$ & $\mathrm{mJ} \mathrm{mol}^{-1} \mathrm{~K}^{-2}$ & 18.1 & 21.4 & 5.8 & 16.5 \\
\hline$\Delta \mathrm{C} / \gamma \mathrm{T}_{\mathrm{c}}$ & & 1.42 & 0.66 & 1.97 & $\sim 1.4$ \\
\hline$\lambda_{\text {ep }}$ & & 0.5 & 0.51 & 0.79 & 0.44 \\
\hline DOS $\left(\mathrm{E}_{\mathrm{F}}\right)$ & states / eV f.u & 5.1 & 3.0 & 1.4 & 4.9 \\
\hline
\end{tabular}
a) This work;
b) ref. 18;
c) ref. $19, \lambda_{\text {ep }}$ calculated assuming $\mu^{*}=0.13$;
d) ref. 13 


\section{Figures}

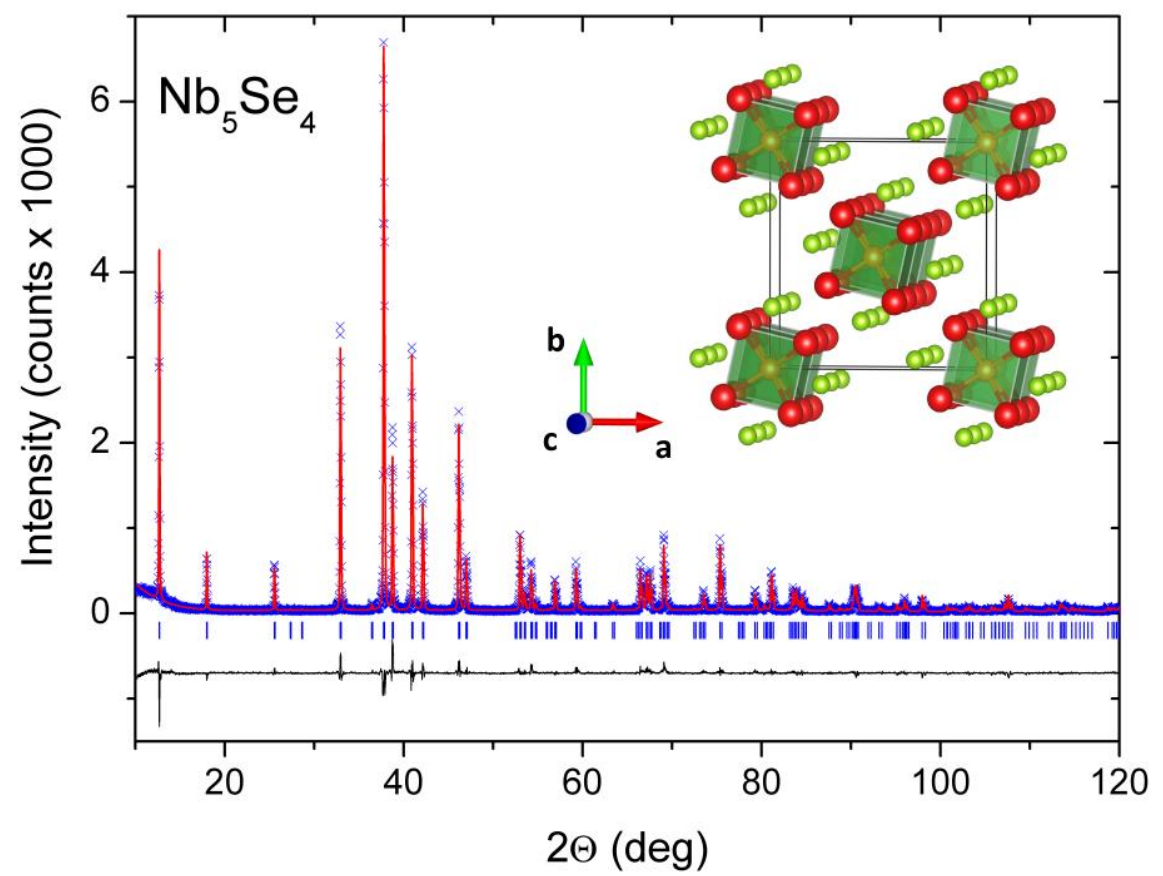

\section{Figure 1}

Rietveld refinement of the room temperature powder X-ray diffraction data for $\mathrm{Nb}_{5} \mathrm{Se}_{4}$. ( $\mathrm{Cu} \mathrm{K} \alpha$ radiation) Observed data and calculated intensity are represented by the crosses and the solid red line, respectively. The difference is shown in the lower part by a solid black line. The blue vertical ticks correspond to the Bragg peaks for $\mathrm{Nb}_{5} \mathrm{Se}_{4}$ : space group I $4 / \mathrm{m}$ (s.g. \# 87), $a=0.985247(23) \mathrm{nm}, c=0.345217(8) \mathrm{nm}$. The inset shows the crystal structure of $\mathrm{Nb}_{5} \mathrm{Se}_{4}$ : the $\mathrm{Nb}$ ions are orange (2a site) and red ( $8 \mathrm{~h}$ site), Se ions are green. 


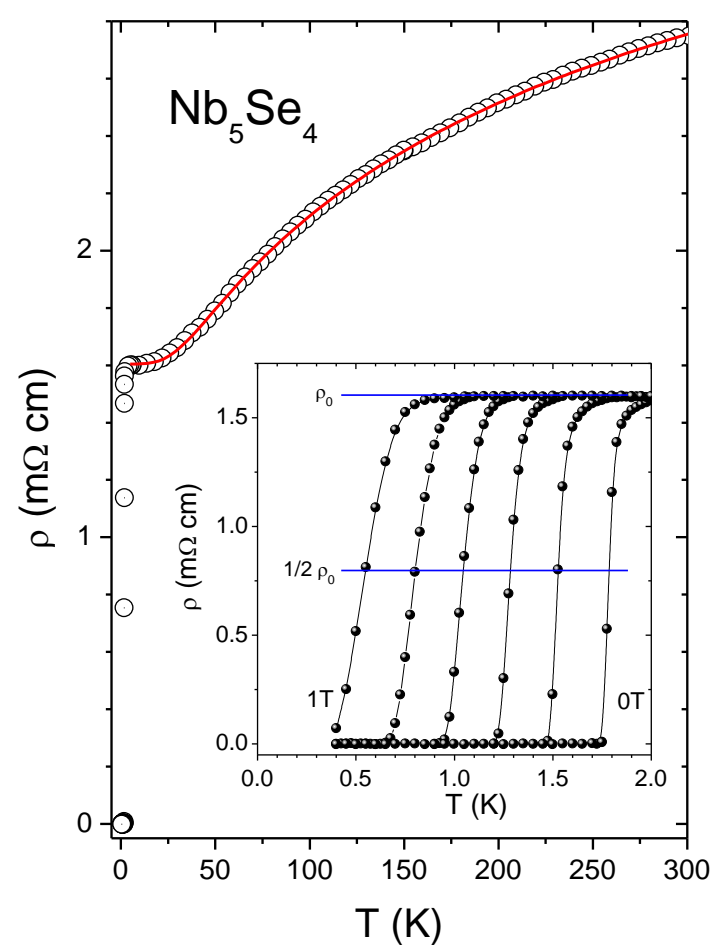

\section{Figure 2}

Characterization of the $\mathrm{Nb}_{5} \mathrm{Se}_{4}$ superconductor through resistivity measurements. Main panel: Electrical resistivity versus temperature for $\mathrm{Nb}_{5} \mathrm{Se}_{4}$ with applied magnetic field $\mu_{0} \mathrm{H}=0 \mathrm{~T}$. The solid line is a fit that combines Bloch Grüneisen resistivity $\rho_{\mathrm{BG}}$ together with a parallel resistor $\rho_{\mathrm{p}}$. Inset: expanded plot of $\rho(\mathrm{T})$ showing the superconducting transition for different values of the applied magnetic field from $\mu_{0} \mathrm{H}=0$ to $1 \mathrm{~T}$. 

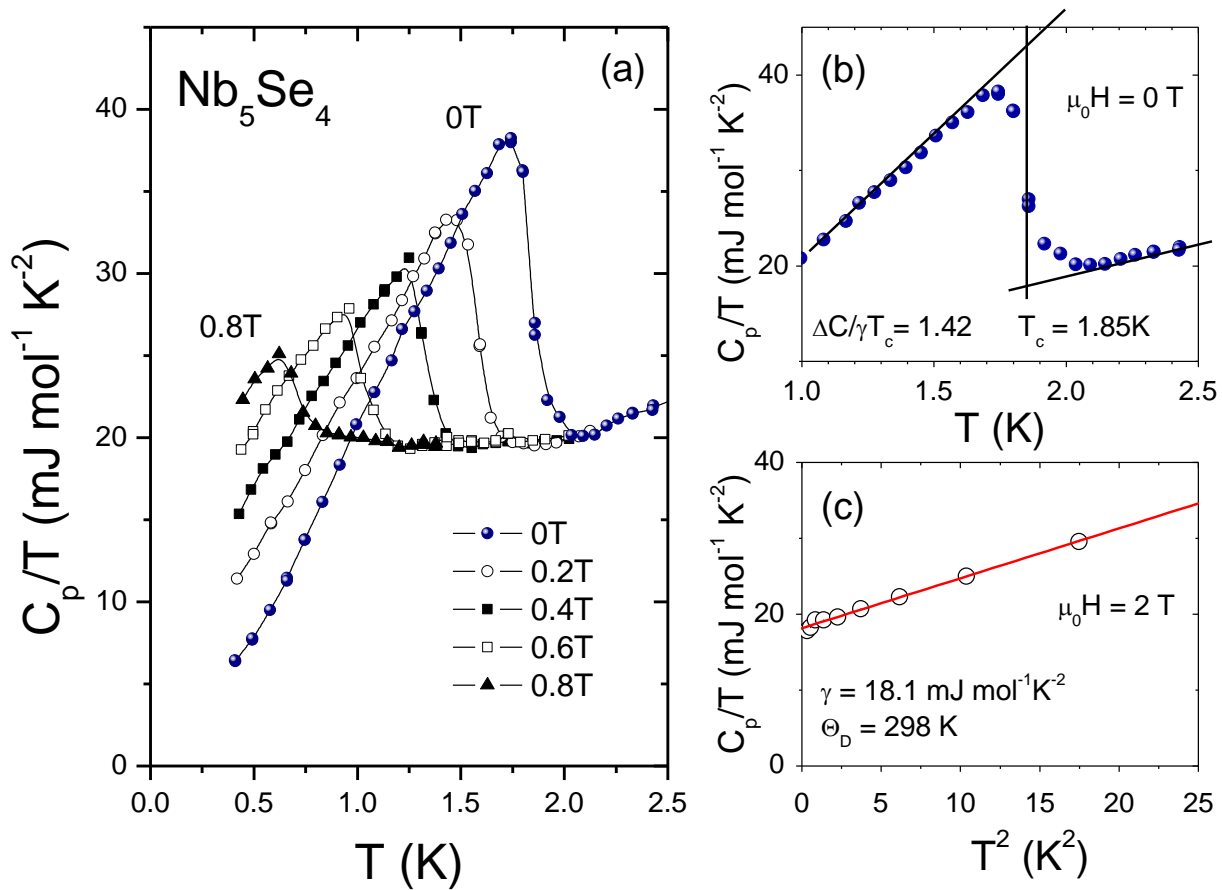

\section{Figure 3}

Characterization of the $\mathrm{Nb}_{5} \mathrm{Se}_{4}$ superconductor through specific heat measurements. (a) Heat capacity plotted as $\mathrm{C}_{\mathrm{p}} / \mathrm{T}$ versus $\mathrm{T}$ between $0.4 \mathrm{~K}$ and $2.5 \mathrm{~K}$ measured with various applied magnetic fields. The solid line in the inset (b) is the entropy conserving construction to estimate the heat capacity jump $\Delta C / T_{c}$ as well as $T_{c}$. (c) $C_{p} / T$ versus $T^{2}$ measured with an applied field of $\mu_{0} \mathrm{H}=2 \mathrm{~T}$. The solid red line is fit by expression $\mathrm{C}_{\mathrm{p}} / \mathrm{T}=\gamma+\beta \mathrm{T}^{2}$. 


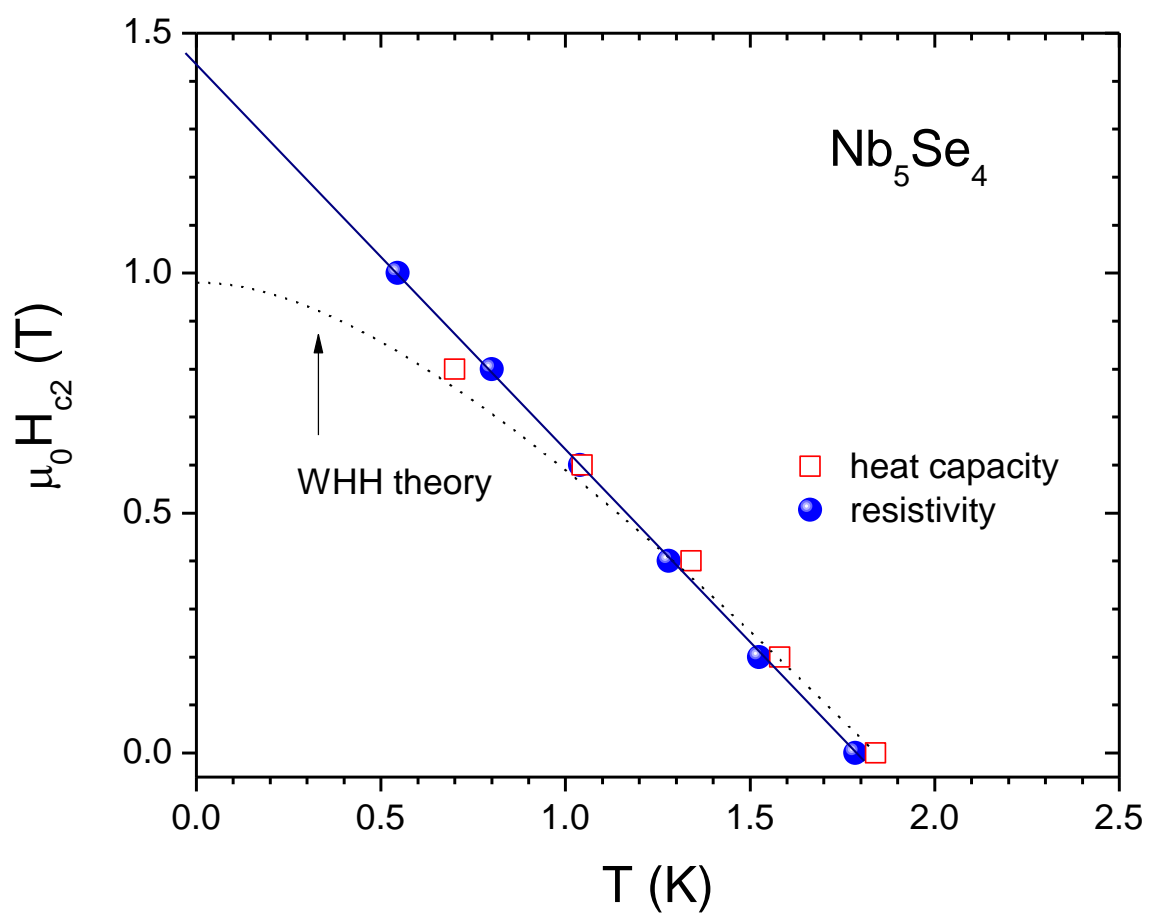

Figure 4.

The temperature dependence of the upper critical field for $\mathrm{Nb}_{5} \mathrm{Se}_{4}$. Open squares and closed circles are data taken from the heat capacity and resistivity measurement, respectively. The solid line shows the linear fit, whereas the dashed line is the fitting curve determined by the WHH formula. 


\section{References}

${ }^{1}$ T. Yokoya, T. Kiss, A. Chainani, S. Shin, M. Nohara, H. Takagi, Science 294, 2518 (2001).

${ }^{2}$ E. Boaknin, M. A. Tanatar, J. Paglione, D. Hawthorn, F. Ronning, R. W. Hill, M. Sutherland, L. Taillefer, J. Sonier, S. M. Hayden, and J.W. Brill, Phys. Rev. Lett. 90, 117003 (2003).

${ }^{3}$ J. Chaussy, P. Haen, J.C. Lasjaunias, P. Monceau, G. Waysand, A. Waintal, A. Meerschaut, P. Molinie and J. Rouxel, Solid State Commun. 20 (1976) 759.

${ }^{4}$ R.M. Fleming, D.E. Moncton and D.W. McWhan, Phys. Rev. B 18 (1978) 5560.

${ }^{5}$ R. Sanjinés, H. Berger, F. Lévy, Materials Research Bulletin 23 (1988) 549-553.

${ }^{6}$ E. Amberger, K. Polborn, M. Dietrich, B. Obst, and P. Grimm, Solid State Commun. 26 (1978) 943.

${ }^{7}$ R.E. Schwall, G.R. Stewart and T.H. Geballe, J. Low Temp. Phys. 22 (1976) 557.

${ }^{8}$ P. Garoche, J.J. Veyssiè, P. Manuel and P. Moliniè, Solid State Commun. 19 (1976) 455.

${ }^{9}$ K. Tsukuma, M. Shimada, M. Koizumi, and H. Kawamura, Phys. Stat. Sol. (a) 65, K1 79 (1981).

${ }^{10}$ H. M. Rietveld, J. Appl. Crystallography 2(2), 65-71 (1969).

${ }^{11}$ J. Rodriguez-Carvajal, FULLPROF: a program for Rietveld refinement and pattern matching analysis, in: Abstracts of the Satellite Meeting on Powder Diffraction of the XV Congress of the IUCr, Tolouse, France, 1990, p. 127.

${ }^{12}$ K. Selte, A. Kjekshus, Acta Chemica Scandinavica (1-27,1973-42,1988) (1963), 17, 2560-2574

${ }^{13}$ A. Stolovits, A. Sherman, R. K. Kremer, Hj. Mattausch, H. Okudera, X.-M. Ren, A. Simon, J. R. O’Brien, Phys. Rev. B 71 (2005) 144519.

${ }^{14}$ T. Klimczuk, C. H. Wang, K. Gofryk, F. Ronning, J. Winterlik, G. H. Fecher, J.-C. Griveau, E. Colineau, C. Felser, J. D. Thompson, D. J. Safarik, and R. J. Cava, Phys. Rev. B 85 (2012) 174505.

${ }^{15}$ A. B. Karki, Y. M. Xiong, N. Haldolaarachchige, S. Stadler, I. Vekhter, P. W. Adams, D. P. Young, W. A. Phelan, and Julia Y. Chan, Phys. Rev. B 83, 144525(2011).

${ }^{16}$ N. R. Werthamer, K. Helfand, and P. C. Hohenberg. Temperature and Purity Dependence of the Superconducting Critical Field, $\mathrm{H}_{\mathrm{c} 2}$. III. Electron Spin and Spin-Orbit Effects. Physical Review 147(1), p. 295 (1966).

${ }^{17}$ V.G. Kogana and R. Prozorov, Rep. Prog. Phys. 75 (2012) 114502.

${ }^{18}$ Hiroyuki Okamoto, Hiromi Taniguti, and Yutaka Ishihara, Phys. Rev. B 53 (1996) 384.

${ }^{19}$ D. Sanchez, A. Junod, J. Muller, H. Berger, F. Lévy, Physica B 204, 167 (1995). 